\title{
Therapeutic Vaccines: A New Revolution
}

\section{Albert Wertheimer}

Temple University, Philadelphia, USA

*Corresponding author: Albert Wertheimer, Temple University, Philadelphia, USA, Tel: +1-215-226-6068; E-mail: albertw@rcn.com

Rec date: Jan 08, 2015; Acc date: Jan 08, 2015; Pub date: Jan 15, 2015

Copyright: (c) 2015 Wertheimer A. This is an open-access article distributed under the terms of the Creative Commons Attribution License, which permits unrestricted use, distribution, and reproduction in any medium, provided the original author and source are credited.

\section{Editorial}

The earliest days of medicines had our ancestors using drugs derived from bark, leaves, stems and other parts of naturally occurring plants. Then in the late 19th century came the heyday of synthetic, organic chemistry which produced nearly all of the "small molecule" medications that we use today. That period lasted over a century and continues today, in 2015 , but there is a powerful contender becoming more prevalent, which has the potential to be the usual therapy of the future. Today, we mainly control symptoms, but do not cure diseases. Examples abound with our current leading drug therapies for diabetes, gout, hypertension, congestive heart failure, COPD, asthma, GERD, glaucoma, ulcers, arthritis and other inflammatory diseases, migraine and a huge array of others.

The therapeutic vaccine era has arrived. There are therapeutic vaccines for myeloma and prostate cancer with others in the pipeline for a growing list of conditions. Now, there is an increasing number of biotechnology products for a diverse array of conditions such as:, paroxysmal nocturnal hemoglobinuria, Maroteaux-Lamy syndrome, cystic fibrosis, hereditary angioedema, multiple sclerosis, chronic myeloid leukemia, multiple myeloma, narcolepsy, advanced colon cancer and many others too numerous to list.

But the most exciting news is that of the therapeutic vaccines. From a list compiled in 2011, there are promising therapeutic vaccines aimed at [1]:

- Glioblastoma

- Celiac disease

- Head and neck cancer

- Cervical cancer

- Pancreatic cancer

- Skin and lung cancer

- Recurrent vulvovaginal candidiasis

- Breast cancer

Surely this list will be increasing rapidly over time. The therapeutic vaccines, unlike the older preventive vaccines, treat a condition or disease by inducing or strengthening the patient's immune response. The first FDA approval of a therapeutic vaccine happened in 2010, ushering in this new therapeutic modality.

One report [2] projects a 55\% sales growth per year for therapeutic vaccines, reaching sales of around US $\$ 13$ billion by 2018 . While several hundred products are in various stages of development, it is not likely that all of them will make it to market. Even if only $25 \%$ are approved for marketing, there is at least 50 new products, many aimed at medical conditions where no cure existed previously. And that estimate of 50 is probably too conservative.

The implications of what therapeutic vaccines may do for patients are probably the subject of dreams for the inflicted patients and their families. However, the impact on pharmaceutical companies could be anywhere from devastating to spectacular depending on individual company strategies. Already, Merck, Pfizer, AstraZeneca, Roche, and Novartis among others have invested in biotechnology companies or bought them outright, or as some have done, licensed biotech products from high technology $\mathrm{R}$ and $\mathrm{D}$ startups that are not prepared or equipped to market them alone.

Companies that have not seen this writing on the wall will find the need for some of their products decline at a precipitous rate. Consider a traditional pharmaceutical company that makes oral medications or injectable insulin for diabetes, only to see new biotherapies that replace damaged pancreatic cells with new, healthy cells that produce insulin effective in managing the body's blood sugar level. Why there would be little or no demand any longer for the majority of current therapies that control disease symptoms without offering any hope or expectation of cure.

Many of the new therapeutic vaccines are aimed at specific cancers. Even if they cured $50 \%$ of those cancers, it is a far cry superior in most cases to the present statistics and the need for treatments that can and do harm other body components would be reduced. Think of the nausea, tiredness and organ damage caused by many chemotherapy regimens or radiation schedules that would be avoided. The pharmacoeconomics people would be thrilled to see the savings in direct payer or insurer costs, and the patients would be happy to not to lose time at work or school and gain productivitythat had been assumed to be lost. That would be especially important and valuable for the self-employed and persons paid an hourly wage.

As with every new technology, the actual results and benefits may not equal $100 \%$ of the expectations, but tweaks, improved products over time and the practitioner community learning the best methods to use the new therapy contribute to outcomes improvements, surely better than today's results in many cases. This writer has very high expectations for this new category of therapeutics.

\section{References}

1. Erickson D (2011) Fierce Vaccines.

2. Oliver Wyman (2012) Therapeutic Vaccines. Health and Life Sciences. 